\section{Benefits of contrast-enhanced ultrasonography for interventional procedures}

\author{
Constantin Arndt Marschner', Johannes Rübenthaler', Matthias Frank Froelich², \\ Vincent Schwarze ${ }^{1}$, Dirk-André Clevert ${ }^{1}$ \\ 'Department of Radiology, University Hospital Munich, Ludwig-Maximilians University, \\ Munich; ${ }^{2}$ Institute of Clinical Radiology and Nuclear Medicine, University Medical Centre \\ Mannheim, Mannheim, Germany
}

For evaluating unclear tumorous lesions, contrast-enhanced ultrasonography (CEUS) is an important imaging modality in addition to contrast-enhanced computed tomography and magnetic resonance imaging, and may provide valuable insights into the microvascularization of tumors in dynamic examinations. In interventional procedures, CEUS can make a valuable contribution in pre-, peri-, and post-interventional settings, reduce radiation exposure and, under certain circumstances, decrease the number of interventions needed for patients.

Keywords: Contrast-enhanced ultrasound; Lesion; Tissue vascularization; Real-time imaging

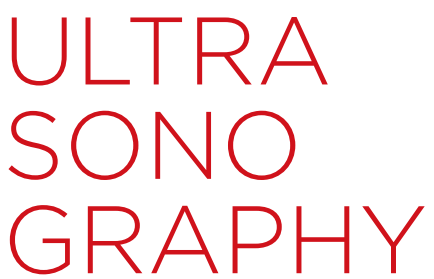

\section{REVIEW ARTICLE}

https://doi.org/10.14366/usg.20083 pISSN: 2288-5919 - elSSN: 2288-5943 Ultrasonography 2021;40:207-216

Received: June 3, 2020

Revised: November 17, 2020

Accepted: November 19, 2020

Correspondence to:

Constantin Arndt Marschner, MD, Department of Radiology, University Hospital Munich, Ludwig-Maximilians University, Marchioninistrasse 15, 81377 Munich, Germany

Tel. +49-89-440073620

Fax. +49-89-440078832

E-mail: constantin.marschner@med. uni-muenchen.de

\section{Introduction}

Contrast-enhanced ultrasonography (CEUS) enables physicians to dynamically assess the vascularization of tissues and vessels in a real-time manner. For this purpose, a contrast agent is injected intravenously, which allows an assessment of the precise contrast dynamics of suspicious lesions. The lesions are evaluated during the arterial phase (15-30 seconds after injection), the portal venous phase (30-70 seconds after injection) and the late phase ( $>70$ seconds after injection) [1-3], which can be transferred as cine loops (5-10 second intervals) to the local memory of the ultrasound unit and subsequently to the local institutional archiving system. The typical malignant characteristics of suspected hepatic metastasis are pronounced early arterial hypervascularization, early wash-out in the portal venous phase, and persistent wash-out in the late phase (Fig. 1). The higher spatial resolution of ultrasound imaging than computed tomography (CT) and magnetic resonance imaging (MRI) enables physicians to safely characterize lesions even smaller than $1 \mathrm{~cm}$, which can be useful for pre-interventional planning, peri-interventional monitoring, and post-interventional treatment response assessment [4].

\section{Overview of the Use of Contrast Media with a Specific Focus on Sonazoid}

The recommended volume of contrast medium varies between 1.0 and $2.4 \mathrm{~mL}$, depending on the

\begin{abstract}
This is an Open Access article distributed under the terms of the Creative Commons Attribution NonCommercial License (http://creativecommons.org/ licenses/by-nc/4.0/) which permits unrestricted noncommercial use, distribution, and reproduction in any medium, provided the original work is properly cited.
\end{abstract}

Copyright (C) 2021 Korean Society of Ultrasound in Medicine (KSUM)

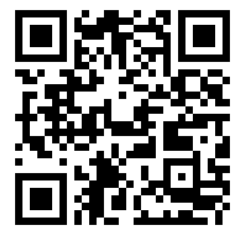

How to cite this article: Marschner CA, Rübenthaler J, Froelich MF Schwarze V, Clevert DA. Benefits of contrastenhanced ultrasonography for interventional procedures. Ultrasonography. $2021 \mathrm{Apr} ; 40(2): 207-$ 216. 
ultrasound system and the tissue to be examined, and is followed by a bolus of up to $10 \mathrm{~mL}$ of sterile saline $(0.9 \% \mathrm{NaCl})[1,5-7]$. Modern contrast agents (e.g., SonoVue, Bracco, Milan, Italy) are small gas-filled microbubbles that generate a nonlinear tissueindependent contrast, allowing dynamic evaluations at the level of the capillary microcirculation with a spatial resolution superior to that of CT and MRI $[4,8,9]$. In order to obtain sufficient imaging quality, a low mechanical index $(<0.2)$ is preferably used to prevent early destruction of the oscillated microbubbles induced by the emitted ultrasound waves. Unlike contrast agents for CT or MRI, which are usually based on iodine or gadolinium, respectively, most ultrasound contrast agents (e.g., SonoVue) have a purely intravascular distribution pattern, which underscores the benefits of CEUS, especially for small lesions [10-13]. In addition, ultrasound contrast agents have extremely low risk profiles and can be administered to a wider range of patients due to their lack of influence on kidney or thyroid gland function $[14,15]$. Early studies demonstrated the safe application of intravenous contrast agents during CEUS examinations in pregnant women $[16,17]$. These agents are also safe for application in children and young adults, and are already widely used in routine practice [18-20].

Sonazoid (GE Healthcare, Waukesha, WI, USA) has unique characteristics as a second-generation ultrasound contrast agent. It was first approved and launched in Japan in 2007 and is currently

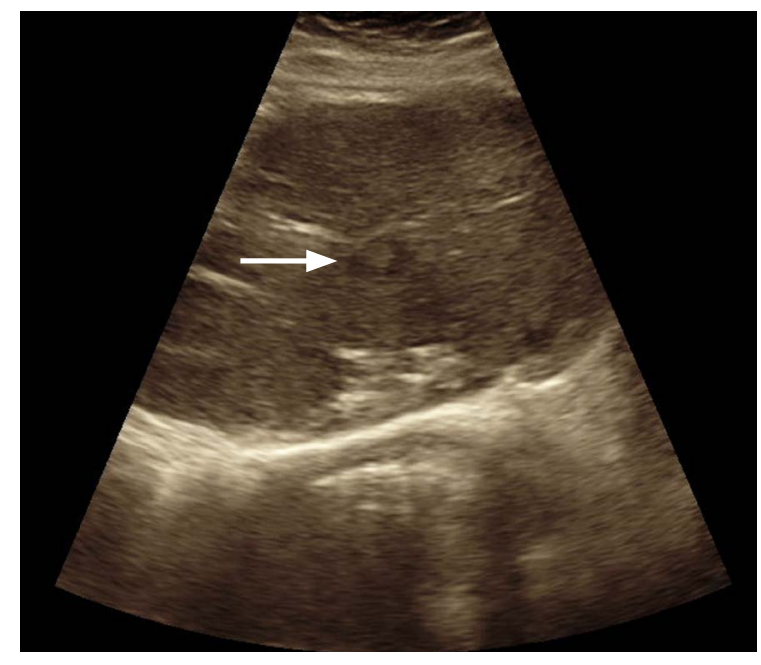

A

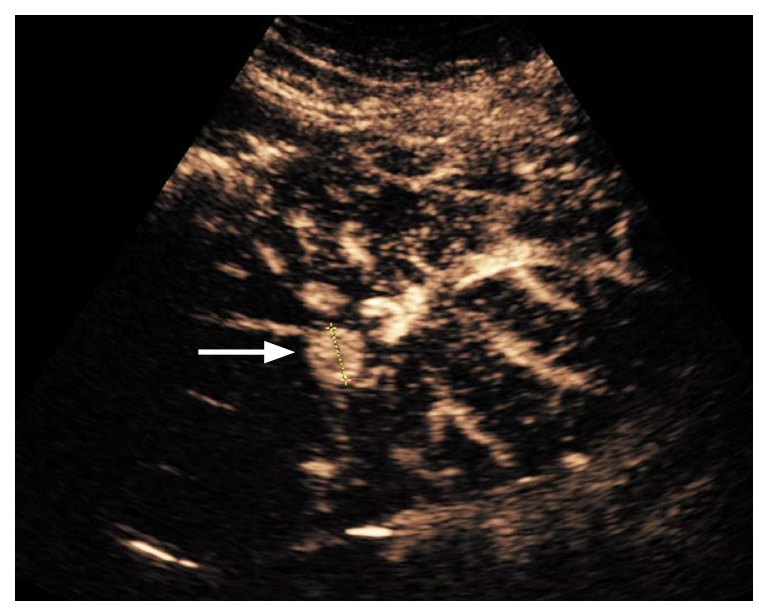

C

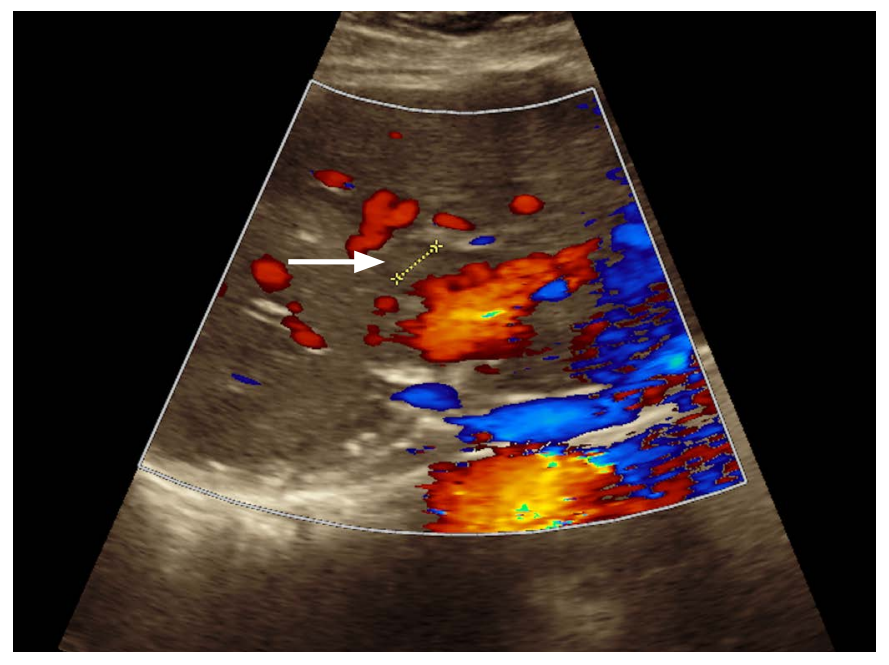

B

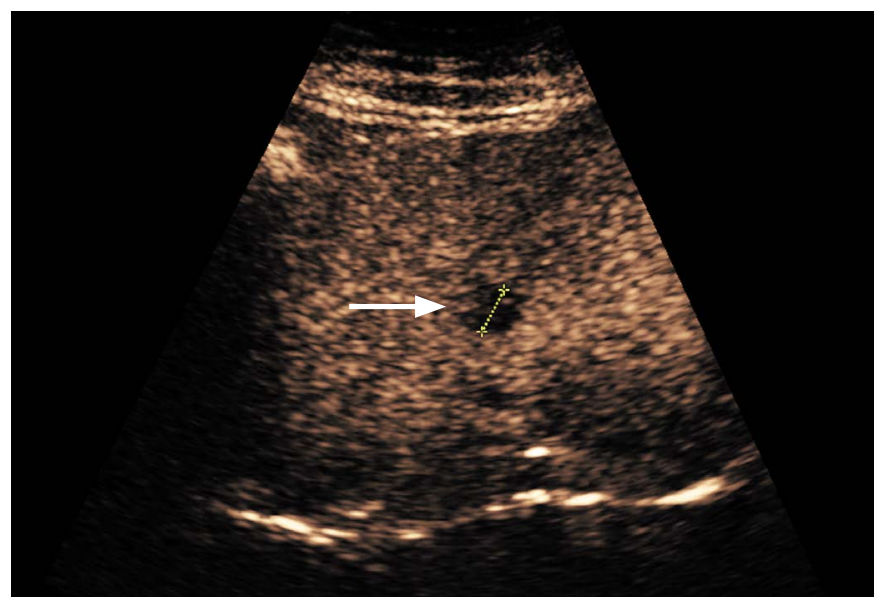

D

Fig. 1. A 54-year-old woman with metastatic colorectal cancer.

A, B. B-mode ultrasonography reveals a 12-mm peripheral hypoechoic lesion (arrow) in liver segment IVa, cranially adjacent to the portal trunk (A) without evidence of hypervascularization (arrow) in duplex sonography (B). C, D. Contrast-enhanced ultrasonography (CEUS) shows an early arterial contrast medium (arrow) (C) with wash-out (arrow) in the portal venous and late phase (D) (5 minutes after injection of contrast medium) indicating a singular liver metastasis. 


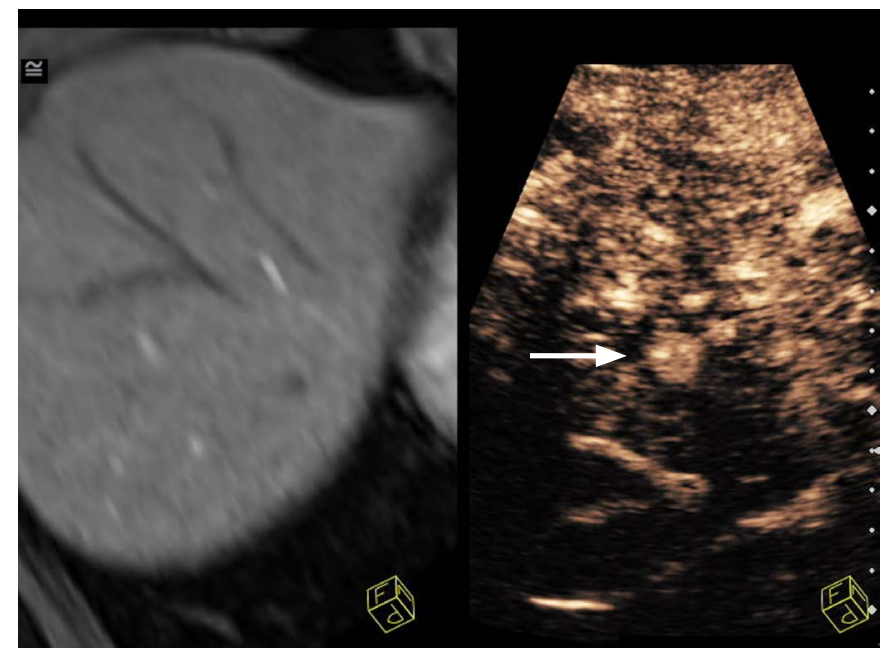

E

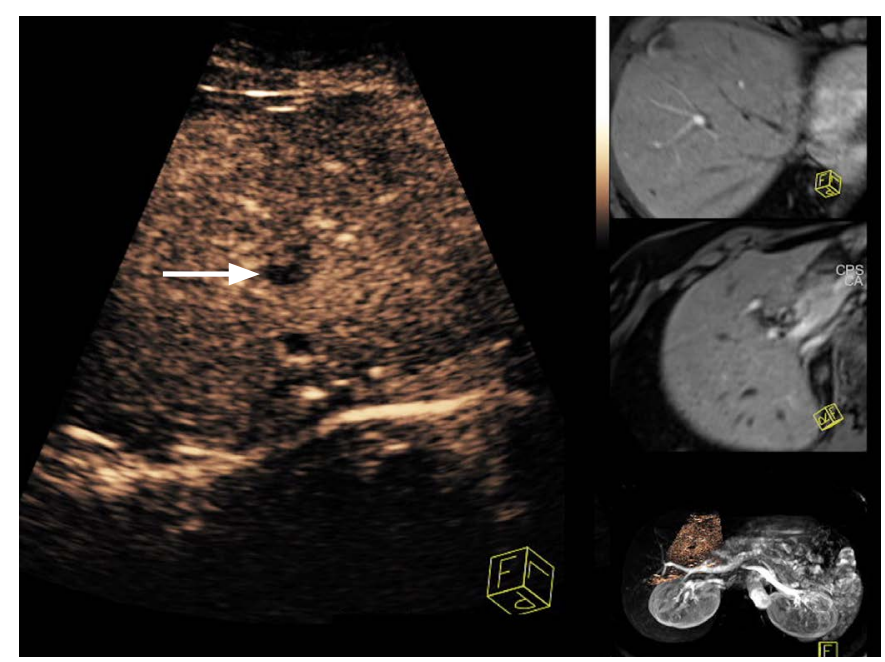

G

also available in Korea, Norway, Singapore, Taiwan, and China. Norway was the first (and currently, the only) country in Europe to approve Sonazoid in 2014. The lack of broad availability can be explained by the number of adverse events (AEs) in early studies. In a prospective study from 2009, AEs were registered in $49.2 \%$ of cases, with fever, nausea, and diarrhea as the three most common clinical symptoms. Adverse drug reactions occurred in $10.4 \%$ of patients; however, all of them were only mild [21]. In a study from 2019 with an investigation period between 2014 and 2015, a markedly lower rate of AEs was described (24.1\%), but this rate is still higher than that of approved contrast media [22].

Sonazoid, a perflubutane-based contrast agent, enables the acquisition of a parenchyma-specific Kupffer phase. The 2- to 3- $\mu \mathrm{m}$ microbubbles are phagocytosed by Kupffer cells, which are liverspecific macrophages, allowing the examiner to evaluate the hepatic parenchymal enhancement for up to 60 minutes [23]. Lesions

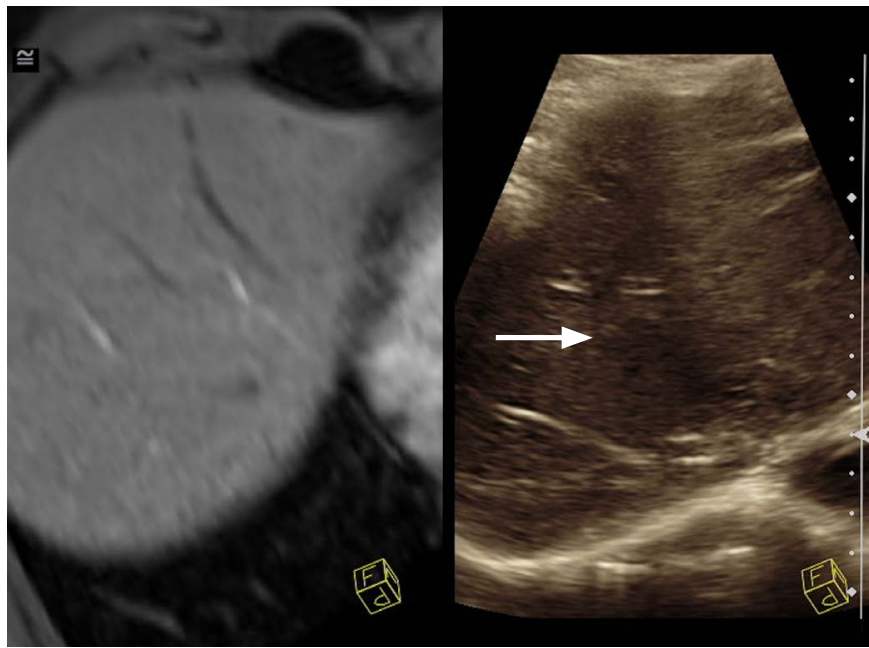

F

Fig. 1. Image fusion of the same patient $(E-G)$ comparing $B$-mode ultrasonography and CEUS to previous magnetic resonance imaging (3 months ago) reveals a new demarcated liver lesions (arrow) with the ultrasonographic and morphological features described above ( $A$, C, D).

such as hepatocellular carcinoma (HCC) or abscesses show no or markedly decreased enhancement in the post-vascular phase due to the lack of regular Kupffer cells [24]. The physician can also generate a defect reperfusion image in which both the arterial and the Kupffer phase can be assessed by repeated administration of Sonazoid in the same slice $[11,25]$. In comparison of their diagnostic value, current studies indicate that Sonazoid is noninferior to established contrast media such as SonoVue [26].

In a consensus statement and recommendation for the clinical practice of contrast-enhanced ultrasound using Sonazoid in 2020, the Asian Federation of Societies for Ultrasound in Medicine and Biology indicated that the further introduction of Sonazoid in the rest of Europe could be expected in the near future due to its low rate of $\mathrm{AEs}(0.5 \%$ and $6.3 \%)$, its advantages regarding a stable time window of up to 60 minutes with a possible improvement of the whole-liver imaging quality, and its benefits within the framework of 
liver interventions [27].

\section{CEUS in the Framework of Liver Interventions}

\section{Puncture and Biopsy}

The advantages of ultrasound-assisted biopsy compared to CT or MRI interventions include the possibility of acquiring realtime imaging, multiplanar image acquisition, and superior costeffectiveness. One of the main benefits of performing CEUSguided punctures or biopsies is the ability to obtain morphological information regarding the microvascular blood supply and blood flow in lesions that could otherwise only be obtained by contrastenhanced CT (CECT) or contrast-enhanced MRI (CEMRI) [27-29]. This advantage enables the physician to differentiate between vascularized and non-vascularized tumor tissue in order to perform a targeted puncture/biopsy and, conversely, to obtain a higher quantity of tissue for histopathological analysis. This fact is highly relevant for partially necrotic lesions, which can be analyzed more accurately than in non-dynamic examinations like CT or MRI. Modern ultrasound devices also provide additional hardware that enables automatic needle guidance to the target lesion, thereby increasing safety, especially for less-experienced examiners [30]. For this purpose, a steering device can be attached to the transducer containing a channel for the needle that can be aligned at different angles. For safe performance of the interventional procedure, it is recommended to first mark the location on the skin, which allows a good access path while avoiding risky structures such as vessels. In comparison to the free-hand technique, the reduced mobility and adjustment of the needle position during the procedure may be regarded as disturbing. Overall, however, there is a significant time advantage with similar success rates, especially for less-experienced examiners [31-33].

In CEUS, the contrast medium can be administered either via peripheral venous access or directly via the inserted needle in order to increase the diagnostic accuracy (e.g., in cystic transformed lesions). In addition, CEUS has a positive impact on diagnostic accuracy in fusion imaging and may be used to safely visualize lesions that are not visible on native B-mode ultrasonography $[34,35]$.

\section{Minimally Invasive Ablative Therapies}

The primary goal of thermal ablation is to induce cell death by intense heat or high radiation induction to achieve devascularization of the suspected lesion with subsequent necrosis. This requires a targeted placement of an ablation probe under imaging guidance, which can, using grayscale ultrasonography, cause difficulties in purely visualized small lesions for even the most-experienced examiners [36]. According to a study by Rim et al., tumors could not be visualized by conventional ultrasonography in 30\% of patients referred for percutaneous radiofrequency ablation (RFA) [37] which underscores the importance and additive value of CEUS.

In the context of interventional therapy planning, CEUS can enable real-time imaging of the topographic anatomy of the lesion to be treated, such as the distance to the surrounding vessels, the liver capsule or other intra- or retro-peritoneal organs such as the stomach, intestine, or kidney. Regarding the latter consideration, peri-interventional insertion of an inflatable balloon could be used to displace the intestines from the radiation field [38]. The possibility of real-time imaging and image fusion can also provide the interventionalist with important information during the intervention $[39,40]$. While conventional B-mode ultrasonography can sometimes be of limited value in the peri-interventional setting, for example due to intralesional gas formation (e.g., during RFA), CEUS can provide additional information if there are contraindications to the use of contrast media for CT or magnetic resonance tomography imaging [41]. In a previous study, the use of Sonazoid led to a significant reduction in RFA sessions when the hepatic lesion was not well-delineated on native B-mode ultrasonography and ablation would have been performed based on the information from CT [42]. Peri-interventional CEUS can also have a positive effect on the detection of residual tumors, which makes it possible to treat these lesions during the same session [43]. However, the investigator must carefully differentiate between residual tumor and peri-ablational hyperemia or gas bubbles at the ablation site in the course of RFA [44].

After the histopathological acquisition of tumorous material and an interdisciplinary decision regarding the implementation of locally invasive therapy, CEUS offers the possibility of a radiationfree procedure to evaluate the success of therapy. Early postinterventional CEUS imaging in combination with preoperative CECT or CEMRI can determine the safety margin after, for example, RFA or transarterial chemoembolization, with a high sensitivity of $80 \%-100 \%$. Thus, CEUS can provide information on the success of local ablative therapy (Fig. 2) [45-47]. Thus, the investigator may be able to preclude tumor recurrence by assessing the lack of revascularization in follow-up examinations [48]. According to the literature, several reports have raised concerns about tumor recurrence after RFA in HCC, the exact underlying mechanism of which is not yet fully understood. One possible explanation is intravascular tumor spread due to a sudden increase of the intracellular pressure in the ablated tissue $[49,50]$. According to the research of Jeong et al. [51], Sonazoid shows a significant suppressive effect on the popping phenomenon, without affecting the clinical outcomes. In general, ultrasound imaging is useful in 


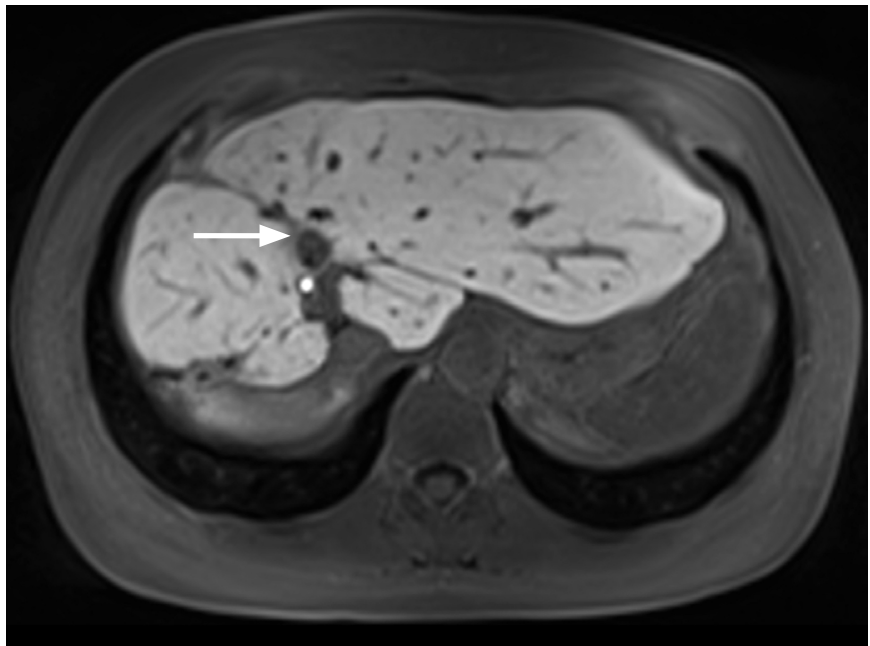

A

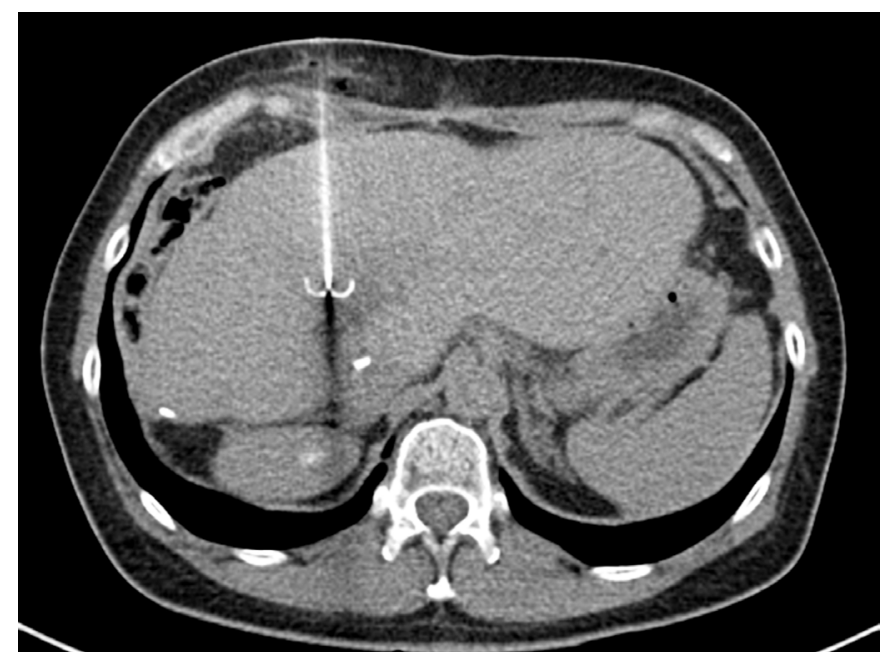

C

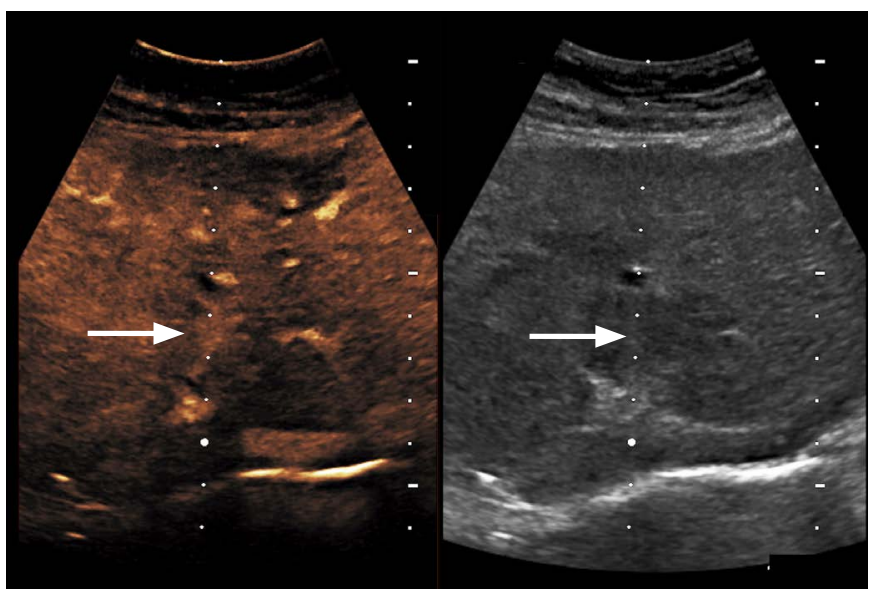

E

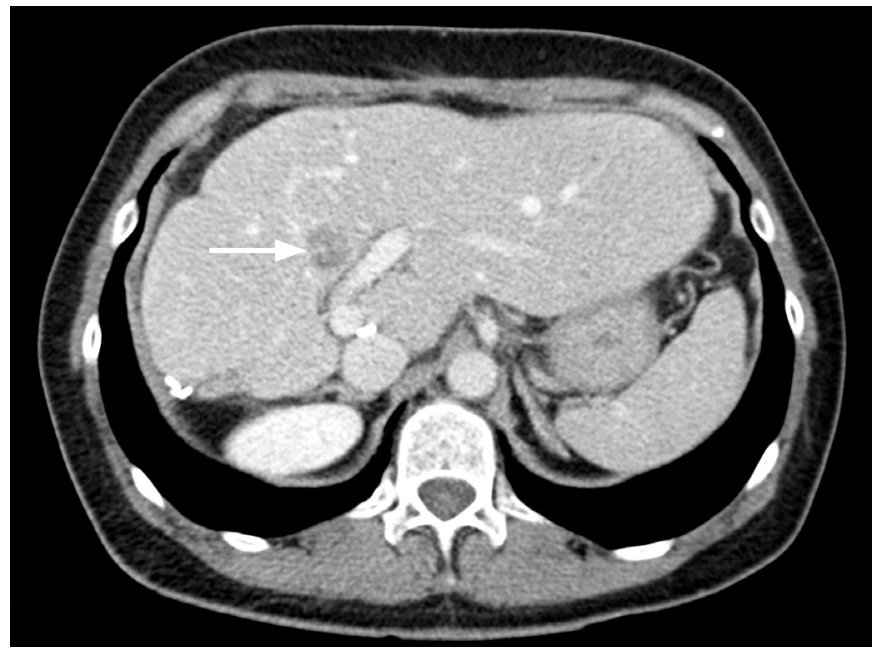

B

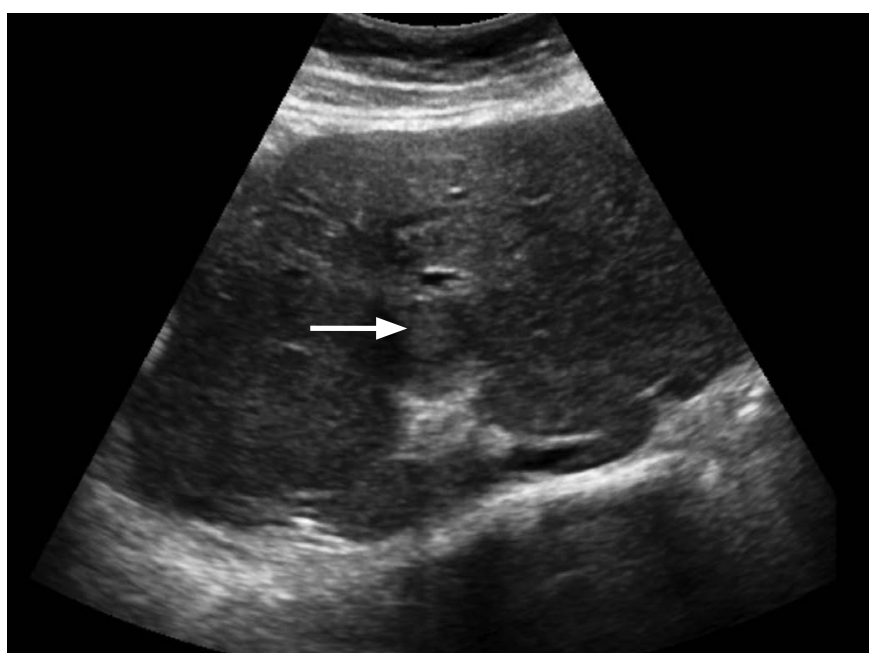

D

Fig. 2. The same patient as in Fig. 1.

A. Magnetic resonance imaging (MRI) shows, similarly to ultrasonography, a liver metastasis in segment IVa with a defect in the hepatobiliary phase (arrow) (contrast agent: Primovist). B, C. On computed tomography, the lesion morphologically shows washout in the venous phase (arrow) (B), which was followed by radiofrequency ablation (RFA) with RFA probe placement (C). D. On B-mode ultrasonography 5 minutes after RFA, the lesion shows a slightly progressive hypoechoic rim (arrow). E, F. In a follow-up examination 5 months after performing RFA, contrast-enhanced ultrasonography (CEUS) shows early arterial enhancement (E) and wash-out (F) at the cranial rim of the treated lesion (arrows). 


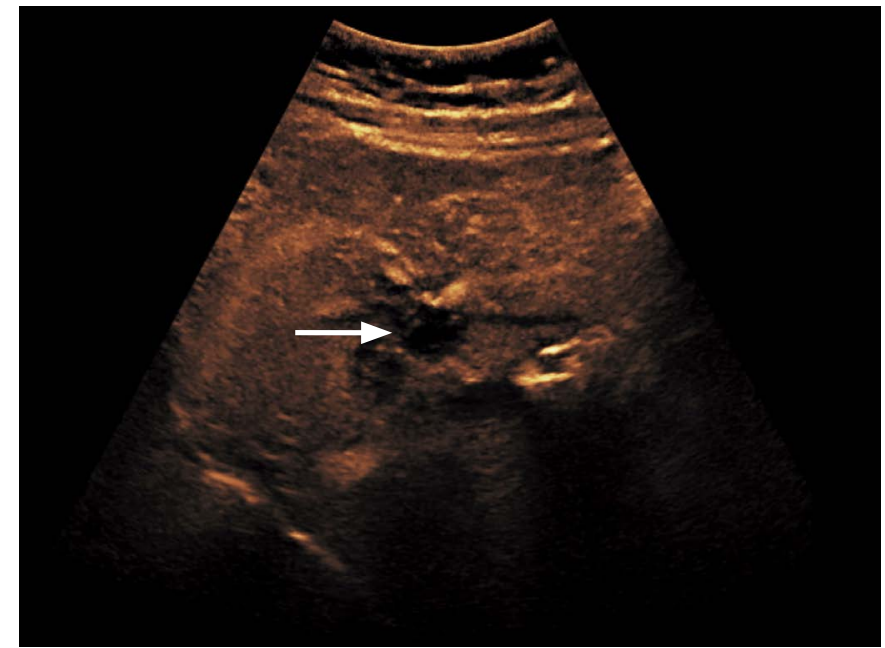

$\mathrm{F}$

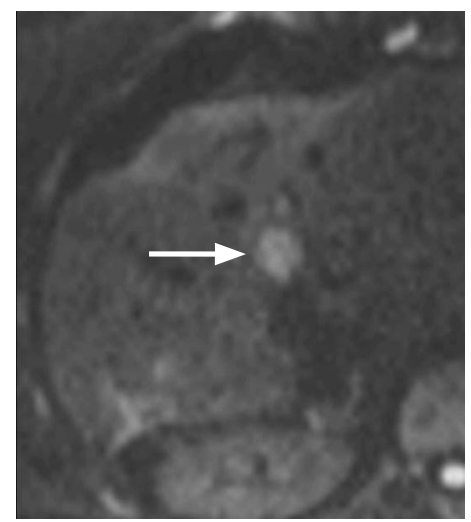

H

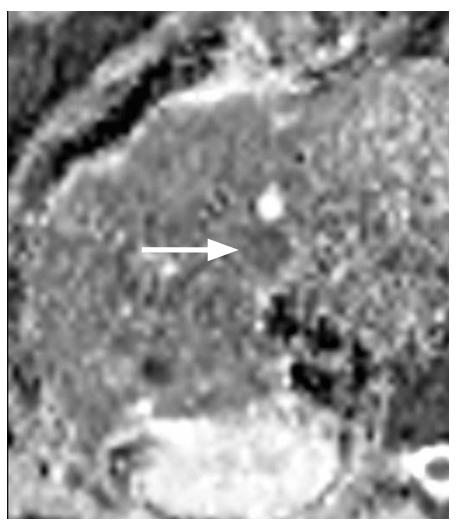

।

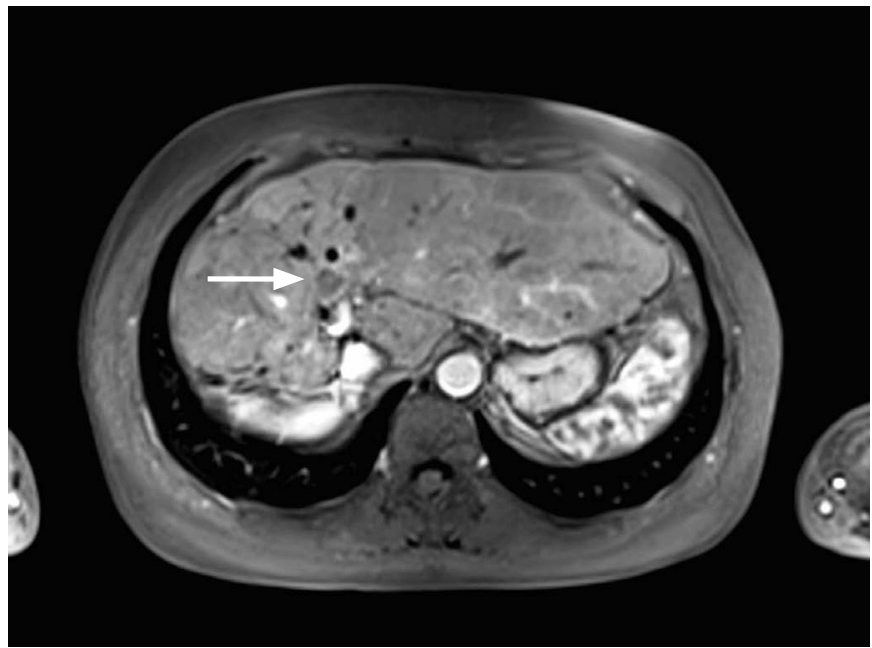

G
Fig. 2. G-I. On MRI, the lesion shows (analogous to CEUS) shallow contrast-medium enhancement $(G)$ with restricted diffusion $(H, I)$, revealing local recurrence (arrows). all pre-, peri-, and post-interventional settings and may provide valuable information on the success of local ablative therapy.

\section{Drainage in Biliary Interventions}

In biliary interventional therapy, CEUS can be utilized for the therapeutic placement of a percutaneous transhepatic biliary drainage (PTBD), which is a common procedure in benign or malignant biliary diseases (Fig. 3). CEUS-PTBD was first described in 2009 and displays typical indications for ultrasound-guided diagnostics [52]. Both native B-mode imaging and CEUS provide high anatomical accuracy in the evaluation of the correct drainage position. However, the field of view can sometimes be limited in native B-mode ultrasonography since an intraluminal tip position can lead to artifacts caused by physiological intestinal gas accumulation. In many cases, additive fluoroscopic cholangiography is required for correct visualization of the catheter tip, which is associated with radiation exposure to the patient. To avoid exposure to ionizing radiation, CEUS can overcome the limitations of native B-mode imaging and thus provide additional information on the drainage position. For this purpose, contrast medium can be applied via the primarily placed drainage, thereby enabling the location of the tip to be directly established in relation to the bile ducts or the small intestine. This is particularly useful if the bile ducts are not dilated or if the question of accidental dislocation of the drainage arises (Fig. 3C) [53]. Another potential complication that can be assessed by CEUS is the presence of a biliary-arterial fistula [54]. In summary, CEUS represents a radiation-free examination technique that can provide valuable information on the correct drainage position and possible complications, and enables the physician to perform the examination at the patient's bedside [36].

\section{Handling of Image Fusion in Ultrasound Imaging}

In order to expand the scope of applications, image fusion has been implemented in modern ultrasound devices. By enabling the sonographer to better visualize small lesions on B-mode ultrasonography, image fusion facilitates pathological clarification 


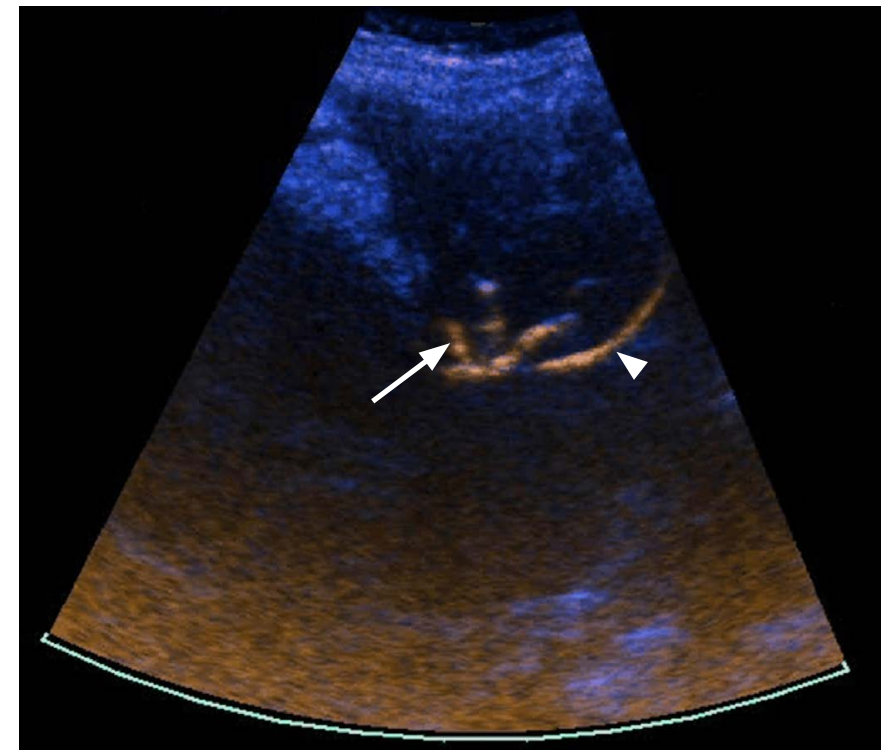

A

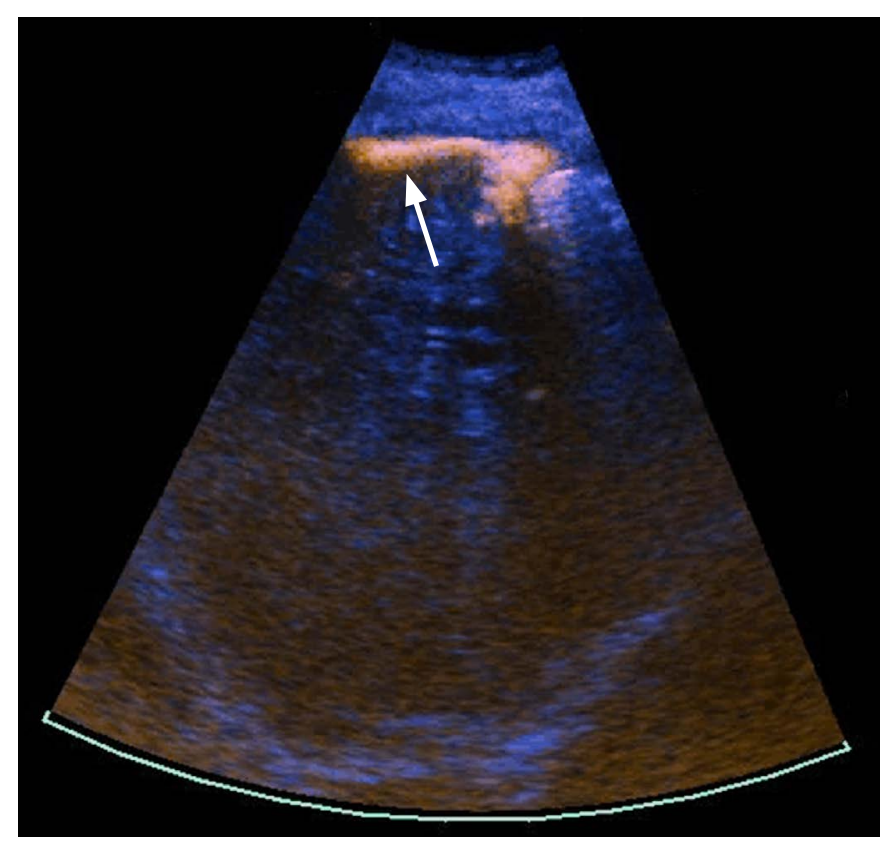

C

or, in the interventional setting, punctures or biopsies of these small lesions $[55,56]$. Further areas of application include postinterventional perfusion analyses for the assessment of local ablative procedures [41].

In the early stages, co-registration in the context of image fusion was considered difficult for inexperienced users, but now runs largely automatically in up-to-date ultrasound machines, often by using a rigid transformation matrix [13]. In the case of manual coregistration, clear and easily found anatomical structures such as large vessels or cysts can be used for better handling. The necessary

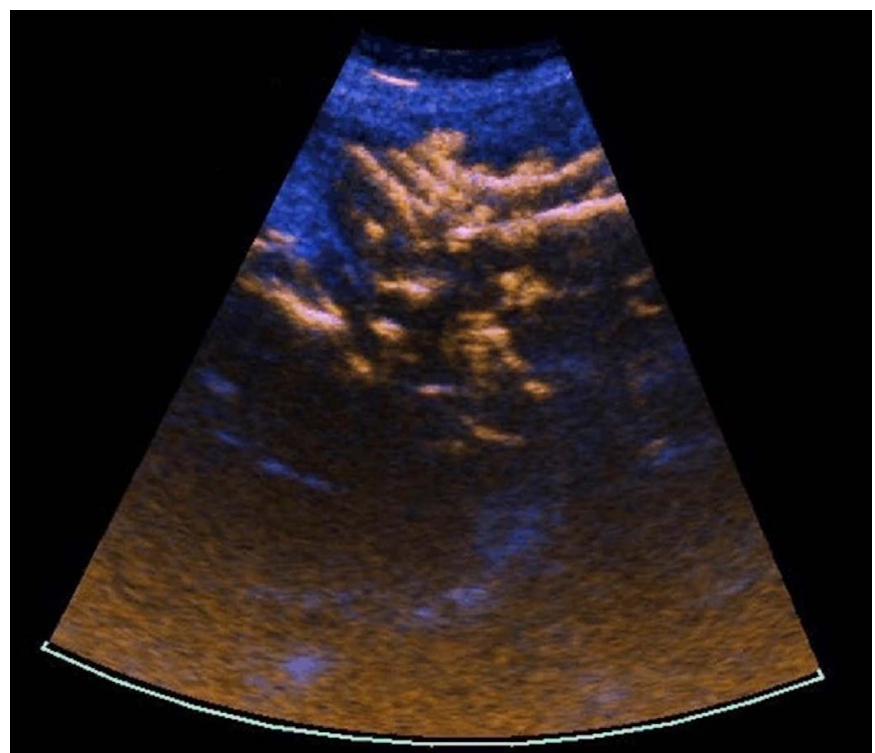

B

Fig. 3. A 62-year-old woman with percutaneous transhepatic biliary drainage (PTBD) (arrowhead).

A. Contrast uptake in the biliary system (arrow, arrowhead) after injection of contrast agent via the drainage, indicates the correct position of the PTBD. After 7 seconds, there is increasing contrast of the central and peripheral bile ducts (B). C. Ultrasonography of the same patient shows displacement of the PTBD after 5 days (arrow).

equipment includes an extra-magnetic field generator and an additional position sensor. The sensor registers the position of the ultrasound probe in interaction with the extra-magnetic field generator and sends the acquired data to the ultrasound device to enable the most accurate co-registration possible. In addition to B-mode imaging, the ultrasound imaging system can also make use of Doppler ultrasonography and CEUS, which covers the entire range of ultrasound imaging and thus allows optimal detection and examination of the microvascularization of the tumorous tissue. The display of the images after successful image fusion can be 
differentiated between a side-by-side mode (Fig. 1E-G), in which the cross-sectional CT or MRI examination is compared to the ultrasound imaging, and an overlay technique, which combines the CT or MRI examination with the currently generated ultrasound examination to bundle the image information from the different examination modalities into one image. Despite its partially automated procedure, image fusion ultrasound examinations require a certain degree of experience in handling the technical precautions and protocols. A disadvantage of the present method is that image fusion adjustment during patient movement or breathing is not always possible to an adequate extent, which may result in ineffective co-registration. Therefore, it is recommended to perform the examination in a breathing position identical to that of the examination used for co-registration.

\section{Conclusion}

CEUS is a diagnostic procedure that can play an important role in planning, performing, and monitoring interventions, as well as in follow-up imaging. Thus, this technique represents an examination with a wide range of possible clinical applications in routine daily practice. CEUS and image fusion combine the general advantages of CEUS imaging with the possibility of combined access to information from additive CT or MRI for detection, therapy, and follow-up.

ORCID: Constantin Arndt Marschner: https://orcid.org/0000-0002-2042-7322; Johannes Rübenthaler: https://orcid.org/0000-0003-0832-5662; Matthias Frank Froelich: https://orcid.org/0000-0001-8501-2147; Vincent Schwarze: https://orcid.org/00000003-3910-7801; Dirk-André Clevert: https://orcid.org/0000-0003-3889-5447

\section{Author Contributions}

Conceptualization: Marschner CA, Rübenthaler J, Clevert DA. Data acquisition: Marschner CA, Rübenthaler J, Schwarze V. Data analysis or interpretation: Marschner CA, Schwarze V, Froelich MF. Drafting of the manuscript: Marschner CA, Schwarze V, Froelich MF. Critical revision of the manuscript: Rübenthaler J, Clevert DA. Approval of the final version of the manuscript: all authors.

\section{Conflict of Interest}

No potential conflict of interest relevant to this article was reported.

\section{References}

1. Dietrich CF, Averkiou M, Nielsen MB, Barr RG, Burns PN, Calliada $F$, et al. How to perform contrast-enhanced ultrasound (CEUS). Ultrasound Int Open 2018;4:E2-E15.

2. Solbiati L, Tonolini M, Cova L, Goldberg SN. The role of contrast- enhanced ultrasound in the detection of focal liver leasions. Eur Radiol 2001;11 Suppl 3:E15-E26.

3. D'Onofrio M, Martone E, Faccioli N, Zamboni G, Malago R, Mucelli RP. Focal liver lesions: sinusoidal phase of CEUS. Abdom Imaging 2006;31:529-536.

4. Strobel D, Bernatik T, Blank W, Schuler A, Greis C, Dietrich CF, et al. Diagnostic accuracy of CEUS in the differential diagnosis of small $(\leq 20 \mathrm{~mm})$ and subcentimetric $(\leq 10 \mathrm{~mm})$ focal liver lesions in comparison with histology: results of the DEGUM multicenter trial. Ultraschall Med 2011;32:593-597.

5. Beyer LP, Wassermann F, Pregler B, Michalik K, Rennert J, Wiesinger I, et al. Characterization of focal liver lesions using CEUS and MRI with liver-specific contrast media: experience of a single radiologic center. Ultraschall Med 2017;38:619-625.

6. Maxeiner A, Fischer T, Schwabe J, Baur ADJ, Stephan C, Peters $\mathrm{R}$, et al. Contrast-enhanced ultrasound (CEUS) and quantitative perfusion analysis in patients with suspicion for prostate cancer Ultraschall Med 2019;40:340-348.

7. Wiesinger I, Wiggermann P, Zausig N, Beyer LP, Salzberger B, Stroszczynski $C$, et al. Percutaneous treatment of malignant liver lesions: evaluation of success using contrast-enhanced ultrasound (CEUS) and perfusion software. Ultraschall Med 2018;39:440-447.

8. Sidhu PS, Cantisani V, Dietrich CF, Gilja OH, Saftoiu A, Bartels $E$, et al. The EFSUMB guidelines and recommendations for the clinical practice of contrast-enhanced ultrasound (CEUS) in nonhepatic applications: update 2017 (long version). Ultraschall Med 2018;39:e2-e44.

9. Negrao de Figueiredo G, Muller-Peltzer K, Rubenthaler J, Clevert DA. CEUS-diagnostic of malignant liver lesions. Radiologe 2018;58:528-537.

10. Ferraioli G, Meloni MF. Contrast-enhanced ultrasonography of the liver using SonoVue. Ultrasonography 2018;37:25-35.

11. Chung YE, Kim KW. Contrast-enhanced ultrasonography: advance and current status in abdominal imaging. Ultrasonography 2015;34:3-18

12. Fischer C, Kunz P, Strauch M, Weber MA, Doll J. Safety profile of musculoskeletal contrast-enhanced ultrasound with sulfur hexafluoride contrast agent. Ther Clin Risk Manag 2020;16:269280.

13. Rubenthaler J, Wilson S, Clevert DA. Multislice computed tomography/contrast-enhanced ultrasound image fusion as a tool for evaluating unclear renal cysts. Ultrasonography 2019;38:181187.

14. Piscaglia F, Bolondi L; Italian Society for Ultrasound in Medicine and Biology (SIUMB) Study Group on Ultrasound Contrast Agents. The safety of Sonovue in abdominal applications: retrospective analysis of 23188 investigations. Ultrasound Med Biol 2006;32:1369-1375.

15. Schwarze V, Marschner C, Negrao de Figueiredo G, Mueller-Peltzer K, Neumann J, Rubenthaler J, et al. SonoVue $®$ does not appear 
to cross the placenta as observed during an examination aimed at confirming a diagnosis of liver echinococcosis in a pregnant woman. Ultraschall Med 2020;41:146-147.

16. Schwarze V, Marschner $C$, Negrao de Figueiredo $G$, Rubenthaler J, Clevert DA. Single-center study: evaluating the diagnostic performance and safety of contrast-enhanced ultrasound (CEUS) in pregnant women to assess hepatic lesions. Ultraschall Med 2020;41:29-35.

17. Schwarze V, Froelich MF, Marschner C, Knosel T, Rubenthaler J, Clevert DA. Safe and pivotal approaches using contrast-enhanced ultrasound for the diagnostic workup of non-obstetric conditions during pregnancy, a single-center experience. Arch Gynecol Obstet 2021;303:103-112.

18. Darge K, Papadopoulou F, Ntoulia A, Bulas DI, Coley BD, Fordham $L A$, et al. Safety of contrast-enhanced ultrasound in children for non-cardiac applications: a review by the Society for Pediatric Radiology (SPR) and the International Contrast Ultrasound Society (ICUS). Pediatr Radiol 2013:43:1063-1073.

19. Ntoulia A, Anupindi SA, Darge K, Back SJ. Applications of contrastenhanced ultrasound in the pediatric abdomen. Abdom Radiol (NY) 2018;43:948-959.

20. Sidhu PS, Cantisani V, Deganello A, Dietrich CF, Duran C, Franke D, et al. Role of contrast-enhanced ultrasound (CEUS) in paediatric practice: an EFSUMB position statement. Ultraschall Med 2017;38:33-43.

21. Moriyasu F, Itoh K. Efficacy of perflubutane microbubble-enhanced ultrasound in the characterization and detection of focal liver lesions: phase 3 multicenter clinical trial. AJR Am J Roentgenol 2009; 193:86-95.

22. Chou YH, Liang JD, Wang SY, Hsu SJ, Hu JT, Yang SS, et al. Safety of perfluorobutane (Sonazoid) in characterizing focal liver lesions. J Med Ultrasound 2019;27:81-85.

23. Luo W, Numata $K$, Kondo M, Morimoto $M$, Sugimori $K$, Hirasawa $\mathrm{K}$, et al. Sonazoid-enhanced ultrasonography for evaluation of the enhancement patterns of focal liver tumors in the late phase by intermittent imaging with a high mechanical index. J Ultrasound Med 2009;28:439-448.

24. Park JH, Park MS, Lee SJ, Jeong WK, Lee JY, Park MJ, et al. Contrast-enhanced US with perfluorobutane (Sonazoid) used as a surveillance test for hepatocellular carcinoma (HCC) in cirrhosis (SCAN): an exploratory cross-sectional study for a diagnostic trial. BMC Cancer 2017;17:279.

25. Kudo M. Hepatocellular carcinoma 2009 and beyond: from the surveillance to molecular targeted therapy. Oncology 2008;75 Suppl 1:1-12.

26. Zhai HY, Liang P, Yu J, Cao F, Kuang M, Liu FY, et al. Comparison of Sonazoid and SonoVue in the diagnosis of focal liver lesions: a preliminary study. J Ultrasound Med 2019;38:2417-2425.

27. Lee JY, Minami Y, Choi BI, Lee WJ, Chou YH, Jeong WK, et al. The
AFSUMB consensus statements and recommendations for the clinical practice of contrast-enhanced ultrasound using Sonazoid. Ultrasonography 2020;39:191-220.

28. Lipnik AJ, Brown DB. Image-guided percutaneous abdominal mass biopsy: technical and clinical considerations. Radiol Clin North Am 2015;53:1049-1059.

29. Sheafor DH, Paulson EK, Simmons CM, DeLong DM, Nelson RC. Abdominal percutaneous interventional procedures: comparison of CT and US guidance. Radiology 1998;207:705-710.

30. Lee MW. Fusion imaging of real-time ultrasonography with CT or MRI for hepatic intervention. Ultrasonography 2014;33:227-239.

31. Phal PM, Brooks DM, Wolfe R. Sonographically guided biopsy of focal lesions: a comparison of freehand and probe-guided techniques using a phantom. AJR Am J Roentgenol 2005; 184:16521656.

32. Bluvol N, Kornecki A, Shaikh A, Del Rey Fernandez D, Taves DH, Fenster $A$. Freehand versus guided breast biopsy: comparison of accuracy, needle motion, and biopsy time in a tissue model. AJR Am J Roentgenol 2009;192:1720-1725.

33. Shabana W, Kielar A, Vermani V, Fernandes DD, Antoniscu R, Schweitzer M. Accuracy of sonographically guided biopsy using a freehand versus needle-guided technique: computed tomographic correlation study. J Ultrasound Med 2013;32:535-540.

34. Kang TW, Lee MW, Song KD, Kim M, Kim SS, Kim SH, et al. Added value of contrast-enhanced ultrasound on biopsies of focal hepatic lesions invisible on fusion imaging guidance. Korean J Radiol 2017;18:152-161.

35. Minami T, Minami Y, Chishina H, Arizumi T, Takita M, Kitai S, et al. Combination guidance of contrast-enhanced US and fusion imaging in radiofrequency ablation for hepatocellular carcinoma with poor conspicuity on contrast-enhanced US/fusion imaging. Oncology 2014;87 Suppl 1:55-62.

36. Huang DY, Yusuf GT, Daneshi M, Ramnarine R, Deganello A, Sellars $M E$, et al. Contrast-enhanced ultrasound (CEUS) in abdominal intervention. Abdom Radiol (NY) 2018;43:960-976.

37. Rhim H, Lee MH, Kim YS, Choi D, Lee WJ, Lim HK. Planning sonography to assess the feasibility of percutaneous radiofrequency ablation of hepatocellular carcinomas. AJR Am J Roentgenol 2008;190:1324-1330.

38. Tang $Q$, Zhao F, Yu X, Wu L, Lu Z, Yan S. The role of radioprotective spacers in clinical practice: a review. Quant Imaging Med Surg 2018;8:514-524.

39. Min JH, Lim HK, Lim S, Kang TW, Song KD, Choi SY, et al. Radiofrequency ablation of very-early-stage hepatocellular carcinoma inconspicuous on fusion imaging with B-mode US: value of fusion imaging with contrast-enhanced US. Clin Mol Hepatol 2014;20:61-70.

40. Makino $Y$, Imai $Y$, Igura $T$, Ohama $H$, Kogita $S$, Sawai $Y$, et al. Usefulness of the multimodality fusion imaging for the diagnosis 
and treatment of hepatocellular carcinoma. Dig Dis 2012;30:580587.

41. Jung EM, Clevert DA. Contrast-enhanced ultrasound (CEUS) and image fusion for procedures of liver interventions. Radiologe 2018;58:538-544.

42. Masuzaki R, Shiina S, Tateishi R, Yoshida H, Goto E, Sugioka Y, et al. Utility of contrast-enhanced ultrasonography with Sonazoid in radiofrequency ablation for hepatocellular carcinoma. J Gastroenterol Hepatol 2011;26:759-764.

43. Dohmen T, Kataoka E, Yamada I, Miura K, Ohshima S, Shibuya T, et al. Efficacy of contrast-enhanced ultrasonography in radiofrequency ablation for hepatocellular carcinoma. Intern Med 2012;51:1-7.

44. Lekht I, Gulati M, Nayyar M, Katz MD, Ter-Oganesyan R, Marx M, et al. Role of contrast-enhanced ultrasound (CEUS) in evaluation of thermal ablation zone. Abdom Radiol (NY) 2016;41:1511-1521.

45. Bo XW, Xu HX, Guo LH, Sun LP, Li XL, Zhao CK, et al. Ablative safety margin depicted by fusion imaging with post-treatment contrast-enhanced ultrasound and pre-treatment CECT/CEMRI after radiofrequency ablation for liver cancers. $\mathrm{Br} J$ Radiol 2017;90:20170063.

46. Li K, Su Z, Xu E, Huang Q, Zeng Q, Zheng R. Evaluation of the ablation margin of hepatocellular carcinoma using CEUS-CT/MR image fusion in a phantom model and in patients. BMC Cancer 2017;17:61.

47. Sparchez Z, Mocan T, Radu P, Anton O, Bolog N. Contrast enhanced ultrasonography in assessing the treatment response to transarterial chemoembolization in patients with hepatocellular carcinoma. Med Ultrason 2016;18:96-102.

48. Clevert DA, Paprottka PM, Helck A, Reiser M, Trumm CG. Image fusion in the management of thermal tumor ablation of the liver.
Clin Hemorheol Microcirc 2012;52:205-216.

49. Kang TW, Lim HK, Lee MW, Kim YS, Rhim H, Lee WJ, et al. Aggressive intrasegmental recurrence of hepatocellular carcinoma after radiofrequency ablation: risk factors and clinical significance. Radiology 2015;276:274-285.

50. Pua U. Rapid intra-hepatic dissemination of hepatocellular carcinoma with pulmonary metastases following combined locoregional therapy. Korean J Radiol 2013;14:640-642.

51. Jeong DY, Kang TW, Min JH, Song KD, Lee MW, Rhim H, et al. Effect of perfluorobutane microbubbles on radiofrequency ablation for hepatocellular carcinoma: suppression of steam popping and its clinical implication. Korean J Radiol 2020;21:1077-1086.

52. Ignee A, Baum U, Schuessler G, Dietrich CF. Contrast-enhanced ultrasound-guided percutaneous cholangiography and cholangiodrainage (CEUS-PTCD). Endoscopy 2009;41:725-726.

53. Mao R, Xu EJ, Li K, Zheng RQ. Usefulness of contrast-enhanced ultrasound in the diagnosis of biliary leakage following T-tube removal. J Clin Ultrasound 2010;38:38-40.

54. Daneshi M, Rajayogeswaran B, Peddu P, Sidhu PS. Demonstration of an occult biliary-arterial fistula using percutaneous contrastenhanced ultrasound cholangiography in a transplanted liver. J Clin Ultrasound 2014;42:108-111.

55. Rubenthaler J, Paprottka KJ, Marcon J, Reiser M, Clevert DA. MRI and contrast enhanced ultrasound (CEUS) image fusion of renal lesions. Clin Hemorheol Microcirc 2016;64:457-466.

56. Schwarze V, Rubenthaler J, Marschner C, Fabritius MP, Rueckel $J$, Fink $N$, et al. Advanced fusion imaging and contrastenhanced imaging (CT/MRI-CEUS) in oncology. Cancers (Basel) 2020;12:2821. 